\title{
Densificación en los vacíos urbanos del distrito de Buenaventura; Para propender por una ciudad compacta y sostenible
}

\section{Densification in the urban voids of the District of Buenaventura; to promote a compact and sustainable city}

\section{Cómo citar.}

Lerma Bonilla, L. L. (2020). Densificación en los vacíos urbanos del distrito de Buenaventura; Para propender por una ciudad compacta y sostenible. Revista Científica Sabia, 6(1). pp. 149-68. Doi: https://doi.org/10.47366/sabia.v6n1a10

Resumen. Este artículo, pretende demostrar cómo se determinaron y unificaron las densidades urbanas que requiere el Distrito de Buenaventura; para convertirla en una ciudad sostenible, que permita generar más unidades habitacionales. La misma, posee una zona insular altamente densificada contraria a la continental, que tradicionalmente ha tenido una morfología urbana, caracterizada por la dispersión de sus asentamientos; permitiendo la existencia de varios vacíos urbanos periféricos.

Por lo tanto, a través de un proceso matemático, y tomando de base la información estadística de 11 anteproyectos de modelación urbana; se determinaron las densidades propuestas, acordes a las características y normativas de la ciudad. Esto permitió generar 4.400 viviendas más, dentro de estos vacíos urbanos, creando una regeneración urbana que aprovecha toda la infraestructura existente. Sería pertinente, que las autoridades locales consideren estos resultados; para implementarlos en la actualización del Plan de Ordenamiento Territorial.

Palabras claves. Densidades; Regeneración Urbana; Ciudad Sostenible; Ordenamiento Territorial; Normatividad.

Abstrac. This article aims to demonstrate how the urban densities required by the Buenaventura District were determined and unified; to turn it into a sustainable city, which allows generating more housing units. It has a highly densified insular area contrary to the continental one, which traditionally has had an urban morphology, characterized by the dispersion of its settlements; allowing the existence of several peripheral urban voids.

Therefore, through a mathematical process, and based on the statistical information of 11 preliminary urban modeling projects; the proposed densities were determined, according to the characteristics and regulations of the city. This allowed to generate 4,400 more homes, within these urban voids, creating an urban regeneration that takes advantage of all the existing infrastructure. It would be pertinent for the local authorities to consider these results; to implement them in updating the Land Use Plan.

Keywords: Densities; Urban Regeneration; Sustainable City; Territorial Ordering; Regulations. 
Figura 1

Fotos aéreas zona continental de Buenaventura

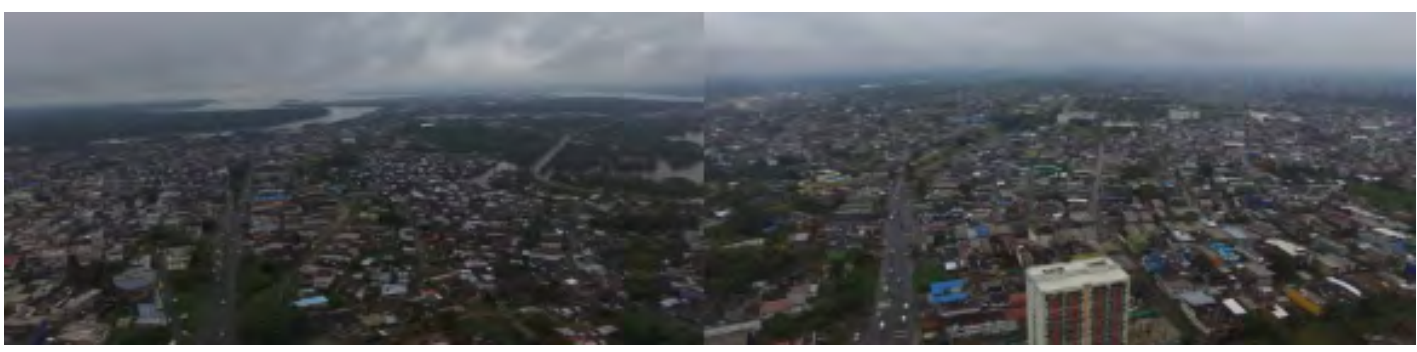

Nota. Fuente: Cuadros, M.A. (2019). Zona continental de Buenaventura. [Fotografía]. https://www. facebook.com/michaelandres.cuadroalvarez/photos

\section{Introducción.}

Desde la mitad del siglo XX, se ha evidenciado la alta densificación de la zona insular de Buenaventura, muy contrario a la baja, presentada en la zona continental (ver Figura 1); que tuvo su desarrollo mediante asentamientos formales e informales, paralelos y dispersos sobre la vía férrea y carretera Simón Bolívar (Botero, 1968), evidenciándose, la génesis del proceso urbanístico de forma lineal de la misma (ver Figura 2). Efecto de desarrollo natural de las ciudades, cuya tendencia de concentración de altas densidades se ubican cercanas a las zonas céntricas y van decreciendo hacia las periferias (Jiménez, 2015); como ha sucedido en Buenaventura, con una Isla de Cascajal altamente densa, ya que desde sus inicios los asentamientos se desarrollaron alrededor del puerto y su centro administrativo (Botero, 1968).

Pero todavía Buenaventura en su zona continental, sigue presentando una morfológica fragmentada, segregada y poco compactada, siendo la peculiaridad de muchas ciudades latinoamericanas (Yunda y Montenegro, 2019); donde los crecimientos son desordenados y en el que su dispersión de crecimiento urbano, supera en proporción a su crecimiento poblacional (Piña, 2018). El desarrollo de este tipo de asentamientos que son poco densas, desordenadas, discontinuas, con grandes vacíos urbanos, falta de equipamientos e infraestructura; es debido a la ausencia de planificación urbana, sobre lo que repercute en los costos de transportes, calidad del espacio público y calidad de vida de sus habitantes (Gómez, 2018).

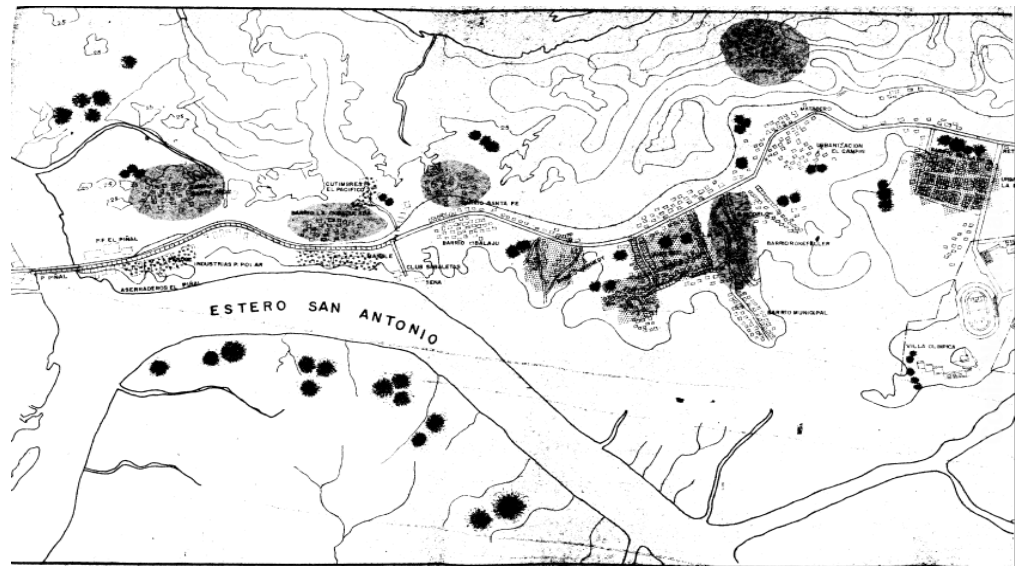

Figura 2. Plano zona continental de Buenaventura entre los sectores del puente del piñal hasta Bellavista en 1968

Nota. Fuente: Corporación Autónoma Regional del Valle del Cauca. Estudio de desarrollo y planeamiento urbano Buenaventura (1968). 
Al contrario, existen ciudades con altas densidades, las cuales tienden a ahorrar energía, incrementan su eficiencia, economizan tiempo, costos y disminuyen el impacto negativo sobre el medio ambiente; siendo una nueva tendencia de modelo urbano, donde además se busca equilibrar el espacio público, localizar bien las áreas residenciales, entre otros aspectos (Ramos, 2017). Aunque muchos argumentan que la densificación es perjudicial para los sistemas naturales, la movilidad y la calidad de vida de la personas (Carrasco, 2020); su resultado arroja en una ciudad, con combinaciones de tejidos urbanos de diferentes densidades (Jiménez, 2015), implementado por la variedades entre estas y los usos del suelo (Zumelsu y Espinoza, 2019), derivando en estructuras urbanas sostenibles y económicamente más eficientes (Treija et al. 2019).

En la actualidad, el crecimiento de muchos asentamientos se han dirigido hacia los lugares menos densos que están alejados de los centros urbanos (Pérez, 2019), tendencia de periferia urbana muy marcada por la influencia de los constructores privados en países latinoamericanos como: México, Brasil y Colombia (Libertun de Duren, 2017), y europeos como: Noruega (Elvestad y Holsen, 2020) y Suiza (Debrunner et al. 2020); donde la expansión urbana posee una tendencia generalizada entre el consumo del suelo y la caída de la densidad (Garcia, 2019), algo ocurrente en los vacíos urbanos periféricos de la zona continental de Buenaventura.

El diseño urbano en el ordenamiento territorial, logra integrar el proceso dado entre la planificación y el edificio; precisamente donde más se evidencian las fallas de planeación urbana (Montrezor y Bernardini, 2019), que es donde se genera, la imagen de ciudad poco densa. Por eso, en la actualidad aparece el concepto de regeneración urbana, como solución de muchas urbes, a enfrentar de manera integral las problemáticas de segregación, desfragmentación, obsolescencia entre otros (Bustos, 2019); concepto que debe contemplarse desde cuatro perspectivas como: la de las políticas, del programas, de procesos y de los proyectos:

"Políticas activas, capaces de implementar programas operativos, y dinamizar procesos integrados implantados en el territorio que se despliegan en varios proyectos. El proyecto de regeneración urbana es el aspecto más cercano a la ciudadanía y al territorio, aunque debe formar parte de un proceso más amplio para la ciudad o región. Las actuaciones de regeneración urbana deben ser entendidas desde una perspectiva integral, pues operan más allá de los objetivos, aspiraciones y conquistas de las reformas urbanas y del desarrollo urbano, y van más allá de la transformación del espacio físico, puesto que deben orientarse hacia la transformación socioeconómica y hacia un cambio en las formas de la gobernanza." (Remesar, 2019, p. 5).

Regeneración urbana, que deberá propender para una ciudad sostenible, que cumpla con los nuevos Objetivos de Desarrollo Sostenible (Gonzales, 2018), donde sus propósitos se alineen con la planificación, la estructura urbana y el sistema de gobierno (Ramos, 2017); por lo que la definición y medición de esta forma sostenible, se ha convertido en un desafío para gobernantes internacionales y locales, planificadores urbanos y arquitectos, a proponer nuevas normativas para la reestructuración y rediseño de las áreas urbanas (Zumelsu y Espinoza, 2019). En este sentido, el abordaje del concepto de sostenibilidad, se hace bajo seis criterios ${ }^{1}$ donde uno de ellos es el de la densidad; criterio sobre el cual, no existe una definición consensuada ni tampoco una regla o parámetro de aplicación de usos y densidades en una zona específica urbana (Zumelsu y Espinoza, 2019), por lo tanto su aplicabilidad depende de las características específicas de cada territorio y a la decisión autónoma de su autoridad.

Por eso resulta importante el concepto de densidad, porque ella siempre hace relación a la forma urbana; pero como un elemento intangible diferente, a las características físicas del espacio urbano o sea diseño urbano (ver Figura 3):

\footnotetext{
1 "Aborda seis criterios de entorno construido relacionados con la sostenibilidad: escala, accesibilidad, conectividad, densidad, diversidad y nodalidad" (Zumelsu \& Espinoza, 2019).
} 
Figura 3. Modelación urbana de vivienda sobre vacíos urbanos: Proyecto $N^{\circ} 1$

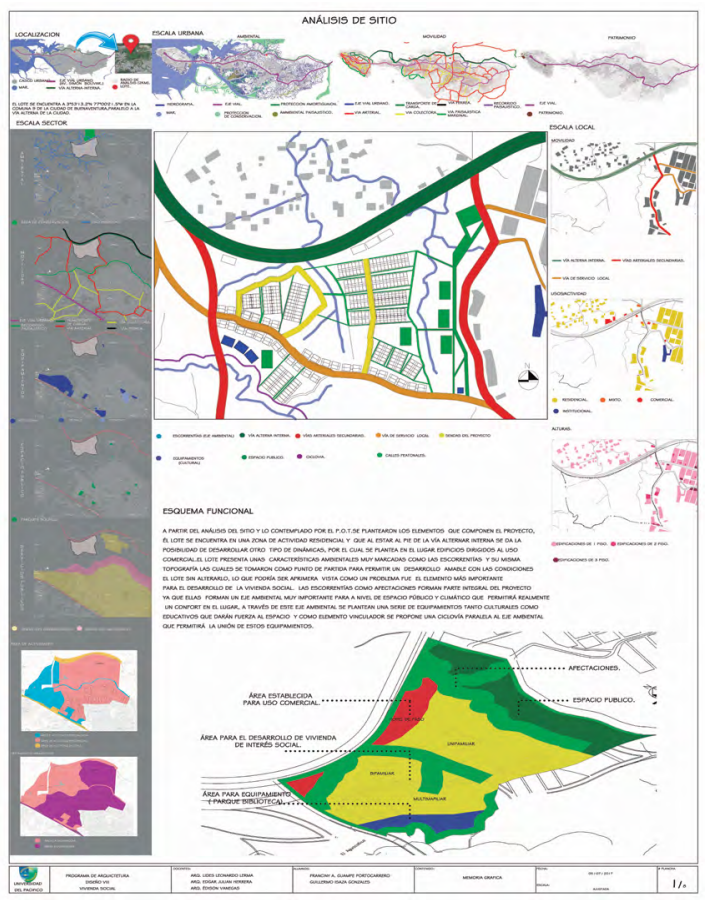

Nota. Fuente: Taller vivienda social - Programa de Arquitectura. Universidad del Pacífico. (2018).

"...la densidad o la nodalidad", que se relacionan a su vez con la configuración del medioambiente social y su interacción dentro de barrios o distritos residenciales. Esta visión integradora entre los usos y usuarios es parte esencial de la noción de forma urbana sostenible en la actualidad". (Zumelsu y Espinoza, 2019, p. 83).

Por lo tanto, al hablar de sostenibilidad urbana, nos referimos paralelamente al concepto de densificación, el cual ofrece ventaja en la planeación de la ciudad porque reduce su consumo de energía, permitiendo mejorar la medición de su sostenibilidad desde su morfología urbana (Ramos, 2017); minimizando el sesgo socio-espacial, mediante el uso de infraestructuras y servicios existentes (Piña, 2018), por lo tanto este concepto ha surgido globalmente como una fuente importante de planeación y desafío político (Dunning et al. 2020). Lográndose además, el acceso y distribución de áreas verdes urbanas de forma equitativa a toda la población, para mejorar su calidad de vida (Sierra, 2018); evitando por supuesto, que los nuevos asentamientos densificados, en el tiempo se conviertan en guetos verticales, si precisamente las regulaciones urbanas no son controladas (Vergara y Asenjo, 2019).

Siendo así, en Latinoamérica ese otorgamiento de planificación urbana se descentralizó a los gobiernos locales, como en el caso de Perú con la Ley 27680 (Granados, 2019), Brasil Ley Federal 10.257/01 (Rodrígues, 2018), y Colombia Ley de ordenamiento territorial (Ley 388 de 1997, 1997); lo que dio como resultado la expedición de los respectivos planes de ordenamientos territoriales, como el de Buenaventura (Acuerdo 03 de 2001 [Concejo Municipal de Buenaventura]. Por el cual se adopta el Plan de Ordenamiento Territorial para el municipio de Buenaventura. 08 de mayo de 2001); resaltando que estos sistemas territoriales de planificación en casi todos los países de la región, han derivado en modelos de desarrollo que conllevaron a desequilibrios e inequidades sociales (Calle et al. 2019).

2 "La Nodalidad. Este elemento, que se interrelaciona fuertemente con temas de escala, se asocia a la idea que el desarrollo urbano debe organizarse en torno a nodos de diferentes niveles y tamaños (Batty, 2005; Frey,1999; Wilson,2000)" (Zumelsu \& Espinoza, 2019). 
El Plan de Ordenamiento Territorial de Buenaventura denominado de ahora en adelante como P.O.T, dispuso diferentes tipos de densidades para las actividades residenciales; que van desde los rangos de 5 (mínima baja) hasta 117 (máxima alta) viviendas por hectárea neta (v/ha) ${ }^{3}$; las cuales se distribuyen en 15 zonas residenciales urbanas, que tendrán usos compatibles o complementarios 0 de aprobación restringida.

Pero tanto a nivel nacional y local, no existen fundamentos teóricos precisos, de cómo se estimaron los diferentes rangos de las densidades urbanas; ni mucho menos su aplicabilidad específica en cada una de las diferentes áreas residenciales de la ciudad. Muy a pesar, que en el siglo XX a nivel mundial existen diferentes modelos de cuantificación; estos no se han aproximado al cálculo detallado de las mismas, debido a la limitante de información (Yunda y Montenegro, 2019); por lo que podemos concluir, que no existe fundamento para el cómputo y aplicación de las densidades para un territorio (Rodríguez y Rivero, 2017).

Basados en el criterio de densificación que determina la ciudad compacta y sostenible, se estableció una metodología fundamentada en los criterios de sostenibilidad (Rodríguez y Rivero, 2017); pero adaptada a las condiciones locativas de Buenaventura, que están determinadas en la normatividad del P.O.T vigente. Teniendo en cuenta que, para la gestión pública de la vivienda de interés social, esta localidad tiene la gran ventaja de contar con bastantes vacíos urbanos (bienes fiscales), propiedad del Distrito de Buenaventura (Ley 185 de 1959); muy a pesar de los problemas de tenencia real, que junto con la incidencia en los costos de urbanismo, representan una limitante dentro de la viabilidad para esta gestión pública (Lerma, 2019).

Entonces, plantear la realización de algunos cambios normativos, como la densificación urbana, podría ayudar a impulsar la gestión de la vivienda social local; ya que, en los pocos vacíos urbanos disponibles, podrían ofertarse más unidades habitacionales por hectáreas de las que actualmente permite la normatividad. Contribuyendo a reducir efectivamente, el déficit cuantitativo, para tener una ciudad más sostenible, mediante el aprovechamiento de los sistemas estructurantes existentes; permitiendo mejorar la movilidad, el espacio público, los equipamientos sociales y tener relación más armónica con el medio ambiente locativo, de características fisiográficas y orográficas particulares (Lerma, 2019), que coadyuven a mejorar también problemas reales de integración e inclusión (Gonzales, 2018).

En la actualidad, el taller de vivienda social de la Universidad del Pacífico, ha desarrollado un proceso de laboratorio de ciudad; mediante la modelación de 11 diseños urbanos de anteproyectos habitacionales sociales, localizados en diferentes localidades de área continental urbana, y que actualmente son vacíos urbanos (ver Figura 4). Estos ejercicios se hacen aplicando la normatividad dada en el P.O.T actual, teniendo en cuenta las desventajas como las condiciones fisiográficas y orográficas de los lugares estudiados; las cuales arrojan pocas áreas netas, que representan, menor número de unidades habitacionales reales para los proyectos. Esto en gran parte determina la viabilidad de los mismos, porque a menor número de viviendas esperadas, menos atractivo sería la factibilidad real del proyecto; en un modelo nacional de gestión, en cabeza del sector privado (Escallón, 2011).

$3 \quad$ Acuerdo 03/2001 - Artículo 156:

- Densidad alta: 70 - $117 \mathrm{v} / \mathrm{ha}$.

- Densidad media: $30-70 \mathrm{v} / \mathrm{ha}$.

- Densidad media alta: $50-70 \mathrm{v} / \mathrm{ha}$.

- Densidad media baja: $30-50 \mathrm{v} / \mathrm{ha}$.

- Densidad baja: 5 - 30 v/ha. 


\section{Figura 4}

Planos de los anteproyectos de modelación urbana para proyectos de vivienda social en la zona continental de Buenaventura

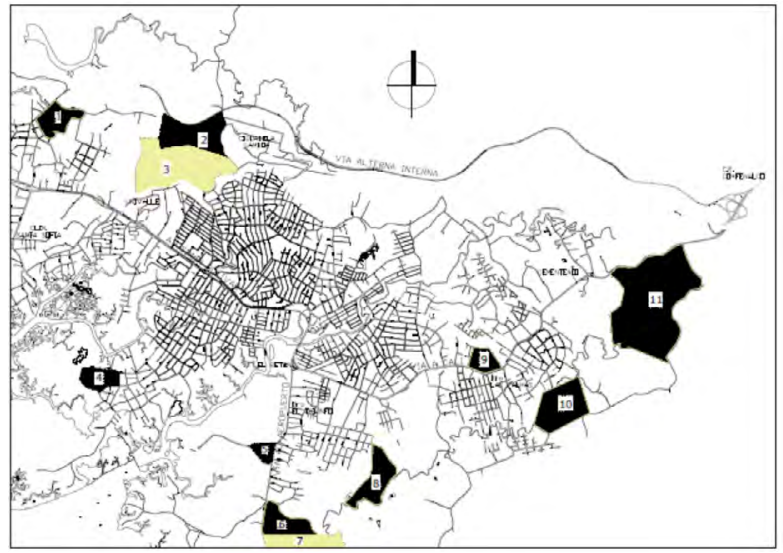

Nota: Fuente: Taller de vivienda social - Programa de Arquitectura. Universidad del Pacífico. (2018). Además, de que estos ejercicios al realizarse en los actuales vacíos urbanos, utilizan los mismos sistemas estructurantes; constituyéndose así una gestión más eficiente, al recurrir a menos recursos que propendan a futuro, a tener una ciudad sostenible. Evitando, que términos como ampliación de infraestructura para cobertura vial y de servicios, sean minimizados en gran proporción; ya que los existentes pueden repotenciarse para cubrir la demanda de los futuros asentamientos de vivienda.

Por lo tanto, el planteamiento hipotético va encaminado en afirmar que, si se aumentan y unifican las actuales densidades, en las existentes zonas residenciales urbanas determinadas en el Plan de Ordenamiento Territorial, la pregunta que surge es:

¿Se lograría incrementar el número de viviendas por hectárea neta, generándose una ciudad compacta y sostenible; mejorándose así los procesos de gestión de proyectos habitacionales? Se pueden definir los siguientes objetivos específicos:

1. Determinar, la deducción del número de vivienda óptima por hectárea, para el Distrito de Buenaventura; estableciendo las densidades máximas, mínimas y promedio propuestas para la ciudad.

2. Establecer, tanto la densidad promedio de los vacíos urbanos existentes, como las áreas netas, según el P.O.T; a través de los resultados establecidos en la modelación de 11 anteproyectos urbanos, de la zona continental de Buenaventura.

3. Estipular, el comparativo del número de unidades habitacionales propuestas, dado entre la densidad promedio existente del P.O.T vigente contra la densidad promedio del estudio propuesto.

\section{Metodología.}

El enfoque metodológico utilizado en este estudio, fue cualitativo de tipo analítico- relacional, mediante la observación de los datos arrojados de la muestra de 11 anteproyectos de vivienda, dentro del proceso de modelación urbana; los cuales mediante un proceso matemático para cálculo de densidades urbanas, permitió generar un modelo especifico de determinación de las ideales para la ciudad de Buenaventura. Estos anteproyectos se localizan en 9 zonas dispersas y aleatorias de la 
franja continental, los cuales son espacios vacíos dentro de un entorno consolidado (ver Figura 5); que adquieren diferentes tipos de densidades según el P.O.T vigente, con usos de suelo destinado para actividad residencial.

El abordaje de cada uno de los tres objetivos específicos señalados, se realizó de la siguiente manera:

\section{Deducción del número de vivienda por hectárea, para determinar las densidades propuestas:}

Para este proceso de cálculo del número de vivienda optimo por hectárea, se tomó como referencia la metodología aplicada en Sevilla -España (Rodríguez y Rivero, 2017), debido a que las otras metodologías solo contemplan los concepto de habitantes y tamaño del territorio (Gómez y Mesa, 2017); la metodología escogida de referencia, tiene la ventaja, de contemplar otras variables como las zonas verdes, superficie vial y plantas construidas, con la cual llegamos a una mejor precisión de resultados.

La superficie urbana ( $S_{\text {urb }}$ ), comprende tres superficies: la superficie construida (Scon), la superficie para zonas verdes $\left(S_{z v}\right)$ y la superficie vial $(S v i a) ; ~ a s i ́:$

\section{Surb=Scon+Szv+Svia (1)}

Considerando que la superficie urbana es una hectárea, o sea $S_{u r b}=10.000 \mathrm{M}^{2}$. Siendo $N_{v}$ es el número de vivienda por hectárea, y $V_{m}$ la superficie media de esas viviendas, expresada en metros cuadrados $\left(\mathrm{M}^{2}\right)$, y $P$ el número de plantas construidas; entonces la superficie construida $\left(S_{\text {con }}\right)$, en metros cuadrados $\left(\mathrm{M}^{2}\right)$ será:

$$
\text { Scon }=\frac{N v \times V m}{P}
$$

Además, considerando que el número promedio de habitantes por vivienda es 3,54; por lo tanto el número de habitantes que habrá en la hectárea es de 3,5 x Nv. La OMS recomienda que la superficie para zonas verdes este en el intervalo de $10-15 \mathrm{M}^{2} / \mathrm{hab}$; donde el P.O.T de Buenaventura plantea que sea $15 \mathrm{M}^{2} / \mathrm{hab}^{5}$ el parámetro deseable, por lo tanto seria el valor a considerar en este trabajo. Entonces superficies para zonas verdes será $S_{z v}=3,5 \times N_{v} \times 15$; o sea $S_{z v}=52,5 N_{v}$

Para calcular la zona víal se considera una calzada de 6 metros de ancho (3 metros en cada dirección) y andenes de 3,5 metros en cada costado ${ }^{6}$ según figura 10 (Anexo 1). Por lo tanto, la superficie víal es $S_{\text {via }}=2.431 \mathrm{M}^{2}$. La expresión queda.

$$
10.000=\frac{N v \times V m}{P}+52,5 N v+2.431
$$

\footnotetext{
${ }^{4}$ DANE 2018. Censo nacional de población y vivienda: En la cabecera municipal de Buenaventura (área urbana) existen 214.833 personas en 61.315 hogares. Por lo tanto, el promedio de personas por hogares de 3,5 hab/viv; superando el promedio nacional de 3,1 hab/viv, y del total del distrito (urbano + rural) que es de 3,47 hab/viv.

${ }^{5}$ Acuerdo 03 de 2001. "Artículo 124. Objetivos: Lograr aliviar el déficit de 14,06 metros cuadrados por habitante de espacio público efectivo, para alcanzar a largo plazo el índice de 15 metros por habitante, en..." ${ }^{6}$ Acuerdo 03 de 2001. Anexo $N^{\circ}$ 5. 36. Dimensión de las vías locales secundarias (V-6) 2.4 Ancho total de la zona vehicular de cada vía (en la parte continental) seis metros (6,00 mts) 2.5 Ancho de las zonas peatonales laterales: ...tres metros con cincuenta centímetros en la parte continental de la ciudad en cada costado (3,50 mts). (Se consideró este factor de medida por ser la mínima contemplada en el ordenamiento territorial).
} 
Entonces:

$$
N v=\frac{7.569}{\frac{V m}{P}+52,5}
$$

A partir de esta expresión, se dequce el numero máximo de viviendas $\left(N_{\mathrm{VM}}\right)$ y el número de viviendas $\left(N_{v m}\right)$, que puede haber en una hectárea. El $N_{v M}$ se obtiene cuando se considera que el número de plantas construidas sea infinito, es decir que la expresión (4), queda:

$$
N v M=\lim _{P \rightarrow \infty} \frac{7.569}{\frac{V m}{P}+52,5}
$$

Es decir, $N_{v M}=144,17$ vivda/ha' . El $N_{v m}$ se obtendrá cuando el número de plantas sea uno, es decir $p$ = 1. La expresión (4), queda:

$$
N v M=\frac{7.569}{\frac{V m}{P}+52,5}
$$

Si $15 \mathrm{M}^{2} / \mathrm{hab}$, es la superficie considerada óptima para zona verde, se considera que la superficie de vivienda va hacer 1,5 veces esa superficie destinada para zona verde; por lo tanto, seria $22,5 \mathrm{M}^{2}$ / hab. Según el Departamento Nacional de Estadísticas - DANE (2018), para Buenaventura el número promedio de habitante por unidad habitacional es 3,5 hab/vivienda; entonces el valor de $V_{m}=78,75$ $M^{2}(22,5 \times 3,5)$. Por eso es que en la expresión (6) al sustituir ese valor el $N_{v m}=57,67 \mathrm{vivda} / \mathrm{ha}^{8}$. El valor optimo ( $\left.N_{v o}\right)$ será el valor promedio de $N_{v m}$ y $N_{v m}$, es decir: $N_{v o}=100,92$ vivda/ha ${ }^{9}$.

Los planteamientos de este trabajo, permiten asignar valores a la superficie de zonas verdes y por último la superficie construida. Con el resultado anterior donde la superficie víal dio, Svia $=2.431 \mathrm{M}^{2}$, que sería un $24,31 \%$ de la superficie urbana residencial. La superficie de la zona verde se obtiene mediante la siguiente expresión (7):

\section{Szv $=100,92$ viviendas $\times 3,5$ hab/vivienda x $15 \mathrm{M} 2 / \mathrm{hab}(7)$}

Es decir que la superficie para zonas verdes será, $S_{z v}=5.298,3 \mathrm{M}^{2}$; equivalentes al $52,98 \%$ de la superficie urbana residencial. Finalmente, la superficie construida ( $\left.S_{c o n}\right)$ para nuestro planteamiento sería, $S_{\text {con }}=2.270,7 \mathrm{M}^{2}$, equivalente al $22,70 \%$. Finalmente, este procedimiento de deducción del número de vivienda por hectárea; permitió generar de una forma más detallada y precisa, las diferentes densidades, que para este estudio se propondrán.

\section{Cálculo del promedio real de densidad y área neta:}

Para este proceso, se tuvo en cuenta los resultados, de la modelación de 11 anteproyectos urbanos de vivienda de interés social ${ }^{10}$ según Tabla $\mathrm{N}^{\circ} 3$ (anexo 2); ubicados en diferentes sitios del área continental urbana del Distrito de Buenaventura. Estos totalizan 235 hectáreas brutas, representando aproximadamente el $65 \%$ de los vacíos urbanos existentes; siendo una muestra significativa para este estudio, que se detalla en la siguiente tabla:

\footnotetext{
${ }^{7}$ Este resultado $N_{v M}$ es equivalente a la densidad máxima propuesta.

${ }^{8}$ Este resultado $N_{v m}$ es equivalente a la densidad mínima propuesta.

${ }^{9}$ Este resultado $N_{v o}$ es el equivalente a la densidad promedio propuesta.

${ }^{10}$ Universidad del Pacífico, Programa de Arquitectura. Diseño VII - Taller vivienda social. (2018).
} 


\begin{tabular}{|c|c|}
\hline 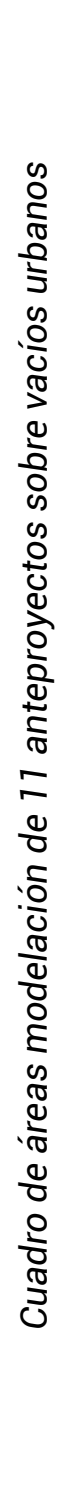 & 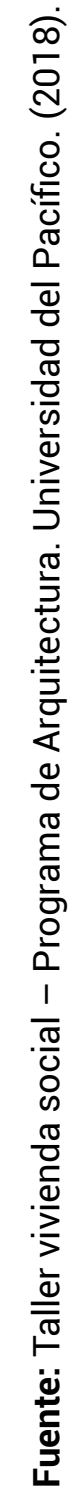 \\
\hline $\begin{array}{l}0 \\
\mathbf{z} \\
\frac{\pi}{0} \\
\frac{0}{\sigma}\end{array}$ & 2 \\
\hline
\end{tabular}

$\circ$

ㅇํำ

く ஜํㅇㅇ

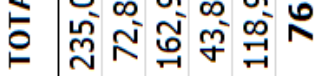

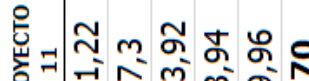

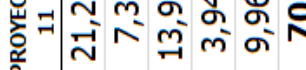

안

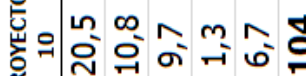

\%

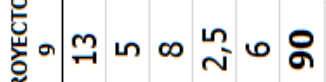

$\circ$

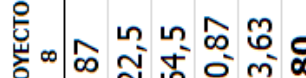

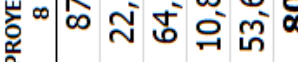

인

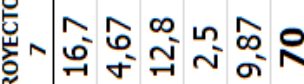

竞

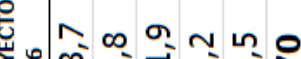

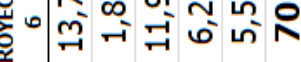

눈

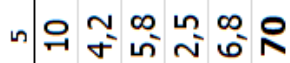


Estos anteproyectos tienen diferentes tipos de densidades, donde 70 vivda/ha es el factor más utilizado según el P.O.T; correspondiente al rango máximo de la densidad media y media alta o al rango mínimo de la densidad alta. Este cuadro también logró determinar las áreas netas; que sobre las 235 hectáreas (100\%) brutas de la modelación, comprobaron finalmente la superficie real disponible para la construcción de los proyectos habitacionales.

\section{Comparativo del potencial de número de viviendas propuestas:}

Para este comparativo finalmente, se cruzó la información de las densidades reales del P.O.T, es decir la máxima de 117 vivda/ha, mínima de 70 vivda/ha y promedio de 76 vivda/ha; contra las densidades propuestas en el cálculo deducción del número de vivienda. Todo, en relación a las áreas netas y útiles, arrojadas la tabla anterior, así: 


\begin{tabular}{|c|c|}
\hline 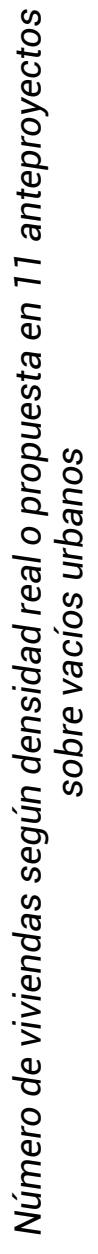 & 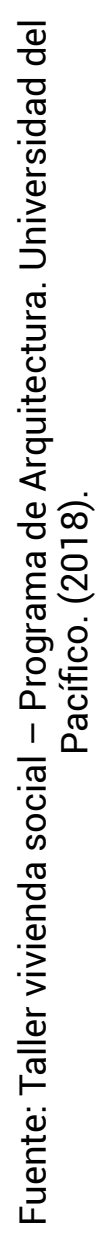 \\
\hline $\begin{array}{l}N \\
0 \\
z \\
\frac{\pi}{0} \\
\frac{\pi}{\pi} \\
1\end{array}$ & $\begin{array}{l}\stackrel{\pi}{0} \\
\mathbf{2}\end{array}$ \\
\hline
\end{tabular}

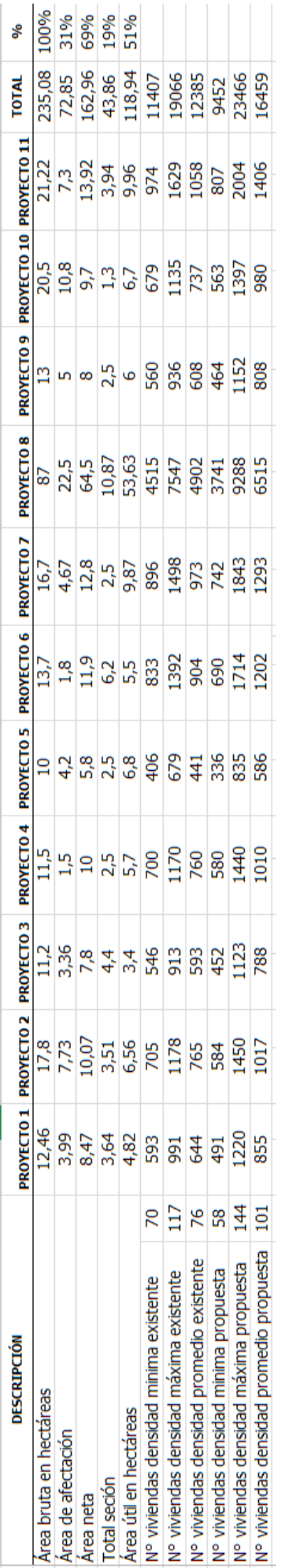


Densificación en los vacíos urbanos del distrito de Buenaventura;

Para propender por una ciudad compacta y sostenible

Lerma Bonilla, Lides Leonardo

Figura 5. Vista aérea de localización de los anteproyectos de modelación urbana en Buenaventura

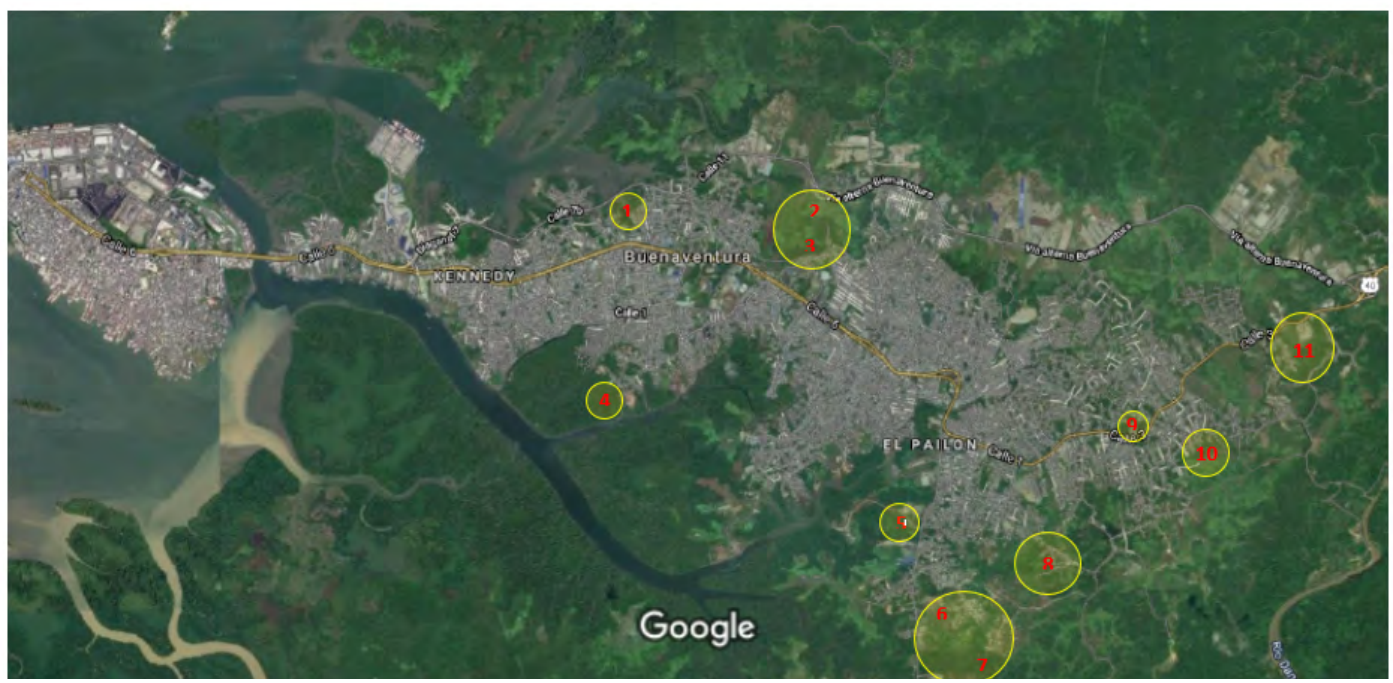

Nota: Fuente: Imágenes @2020 CNE / Airbus, Landsat / Copernicus, Maxar Technologies, U.S. Geological Survey, Datos del mapa @2020.

\section{Resultados y discusión.}

\section{Resultados:}

Según a la metodología planteada en el presente estudio, los resultados obtenidos fueron los siguientes:

- Densidad máxima propuesta en el estudio para el Distrito de Buenaventura, en los vacíos urbanos; con uso para actividad residencial: 101 vivda/ha.

- Densidad máxima propuesta en el estudio, en los vacíos urbanos; con uso para actividad residencial: 58 vivda/ha.

- Densidad máxima propuesta en el estudio, en los vacíos urbanos; con uso para actividad residencial: 144 vivda/ha.

- Área neta promedio de los vacíos urbanos modelados, partiendo de 235 hectáreas brutas (100\%); como referencia total: 162,95 hectáreas (69\%).

- Área de afectación promedio de los vacíos urbanos modelados, partiendo de 235 hectáreas brutas (100\%); como referencia total: 72,85 hectáreas (31\%).

- Número de viviendas, según la densidad mínima planteada actualmente por el P.O.T (70 viv$\mathrm{da} / \mathrm{ha})^{11}$; en 235 hectáreas brutas, de los vacíos urbanos modelados $=11.407$ viviendas.

- Número de viviendas, según la densidad mínima actual (117 vivda/ha); en 235 hectáreas brutas, de los vacíos urbanos modelados $=19.066$ viviendas.

- Número de viviendas, según la densidad promedio actual (76 vivda/ha); en 235 hectáreas brutas, de los vacíos urbanos modelados $=12.385$ viviendas.

- Número de viviendas, según la densidad mínima propuesta en este estudio (58 vivda/ha); en 235 hectáreas brutas, de los vacíos urbanos modelados $=9.452$ viviendas.

- Número de viviendas, según la densidad máxima propuesta en este estudio (144 vivda/ha); en 235 hectáreas brutas, de los vacíos urbanos modelados $=23.466$ viviendas.

${ }^{11}$ Esta densidad de 70 viv/hect; puede ser el factor máximo de las densidades media alta y media en algunos de los proyectos estudiados; pero a la vez en otros proyectos puede ser el factor mínimo de la densidad alta. Para este estudio en relación al cuadro $\mathrm{N}^{\circ} 2$ se dio la denominación de densidad mínima. 
- Número de viviendas, según la densidad promedio propuesta en este estudio (101 vivda/ha); en 235 hectáreas brutas, de los vacíos urbanos modelados $=16.459$ viviendas.

\section{Discusión:}

De acuerdo a los resultados obtenidos, en 235 hectáreas brutas con un área neta promedio de $69 \%$, se logró el aumento de 12.385 a 16.459 viviendas (ver Figura 6); evidenciando que las densificaciones de los vacíos urbanos, optimizan efectivamente las pocas zonas urbanas disponibles para nuevas unidades habitacionales (Carrasco, 2020).

Más si en la actualidad, con el factor máximo de densidad de 117 vivda/ha, se tiene la posibilidad de ejecutar 19.066 viviendas; con el proceso planteado, se llegó a un total de 23.466 unidades habitacionales (ver Figura 7), lográndose un incremento de 4.400 más, dentro del misma área neta disponible de 162,95 hectáreas; donde el simple factor normativo, permitiría esta optimización.

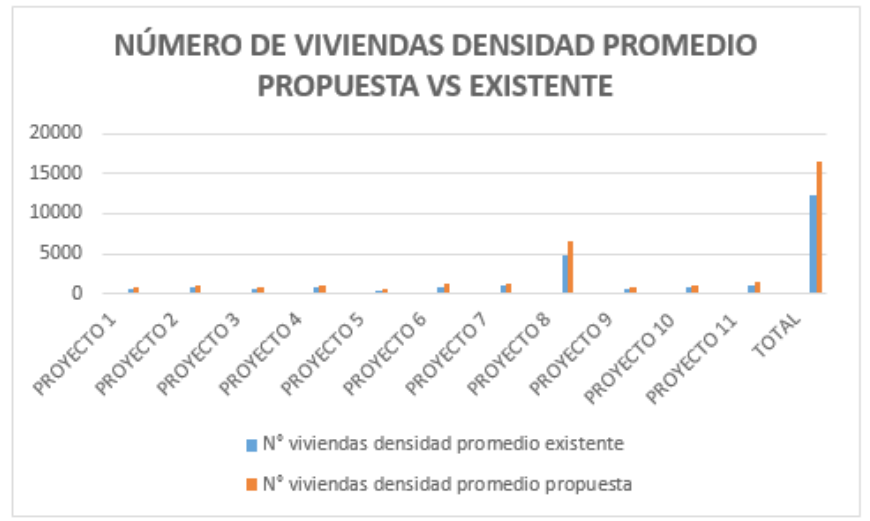

Figura 6. Comparativo de numero de viviendas de densıdad maxıma propuesta contra densidad máxima existente

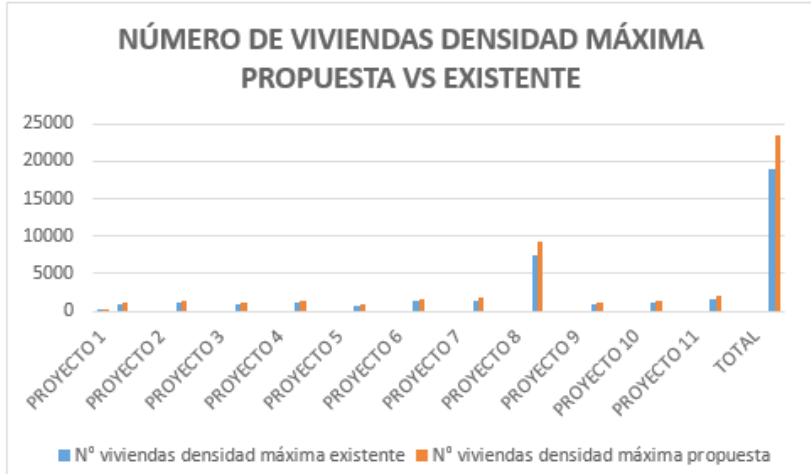

Figura 7. Comparativo de número de viviendas de densidad máxima propuesta contra densidad máxima existente

Más aun, cuando este estudio demostró que solo el promedio de afectación de los lotes de estos vacíos urbanos, están alrededor del $31 \%$; cifra alta que deja en evidencia que, por las condiciones típicas de la fisiografía y orografía de los mismos (Lerma, 2019), sumado a las áreas de cesiones obligatorias (mínimo del 30\% según el P.O.T vigente); el área útil disponible para estos proyectos es del $40 \%$. O sea que, para urbanizar y construir proyectos de vivienda en Buenaventura, siempre quedarán pocas zonas disponibles; por lo que es oportuno compensar esta desventaja, con asentar más unidades habitacionales, de las que actualmente la normatividad permite en determinadas zonas urbanas (Zumelsu y Espinoza, 2019). 
Por lo tanto, las 4.400 viviendas de más que se podrían construir solo en los vacíos urbanos, estudiados en 11 anteproyectos abarcados en 235 hectáreas, significa un incremento aproximado del 23\%; del potencial que estas áreas urbanas podrían generar, para optimizar la oferta de proyectos habitacionales, que coadyuvaría con el déficit cuantitativo en el Distrito. Aun cuando estos vacíos urbanos, se encuentran cerca de la disponibilidad de infraestructura vial y de servicios públicos de la actual trama urbana; lo que significaría un ahorro en recursos, que van a evitar por ejemplo la ampliación de cobertura de la misma, sobre zonas más alejadas (Ramos, 2017).

Representando así, que este ahorro en recursos y optimización de la infraestructura existente, termina siendo una estrategia de regeneración urbana, para una ciudad sostenible (Gonzales, 2018); ya que se aprovecha el entorno del hábitat existente, para alrededor de este implantar uno nuevo, pero utilizando los sistemas estructurantes ya conformados para movilidad, espacio público, equipamiento, servicios públicos y sistema ambiental. Entorno existente, cuya característica morfológica, permite ver un medio urbano poco denso, que consume mucha energía y recursos; terminando por afectar la calidad de vida de la población circundante, representada por ejemplo: en dificultades en la movilidad, baja cobertura y calidad de servicios públicos, deficiencia cuantitativa de espacios públicos y equipamientos sociales (Gómez, 2018); dentro de un hábitat con deficiencias cualitativas (ver Figura 8).

Figura 8. Modelación urbana de vivienda sobre vacíos urbanos: Proyecto $N^{\circ} 1$
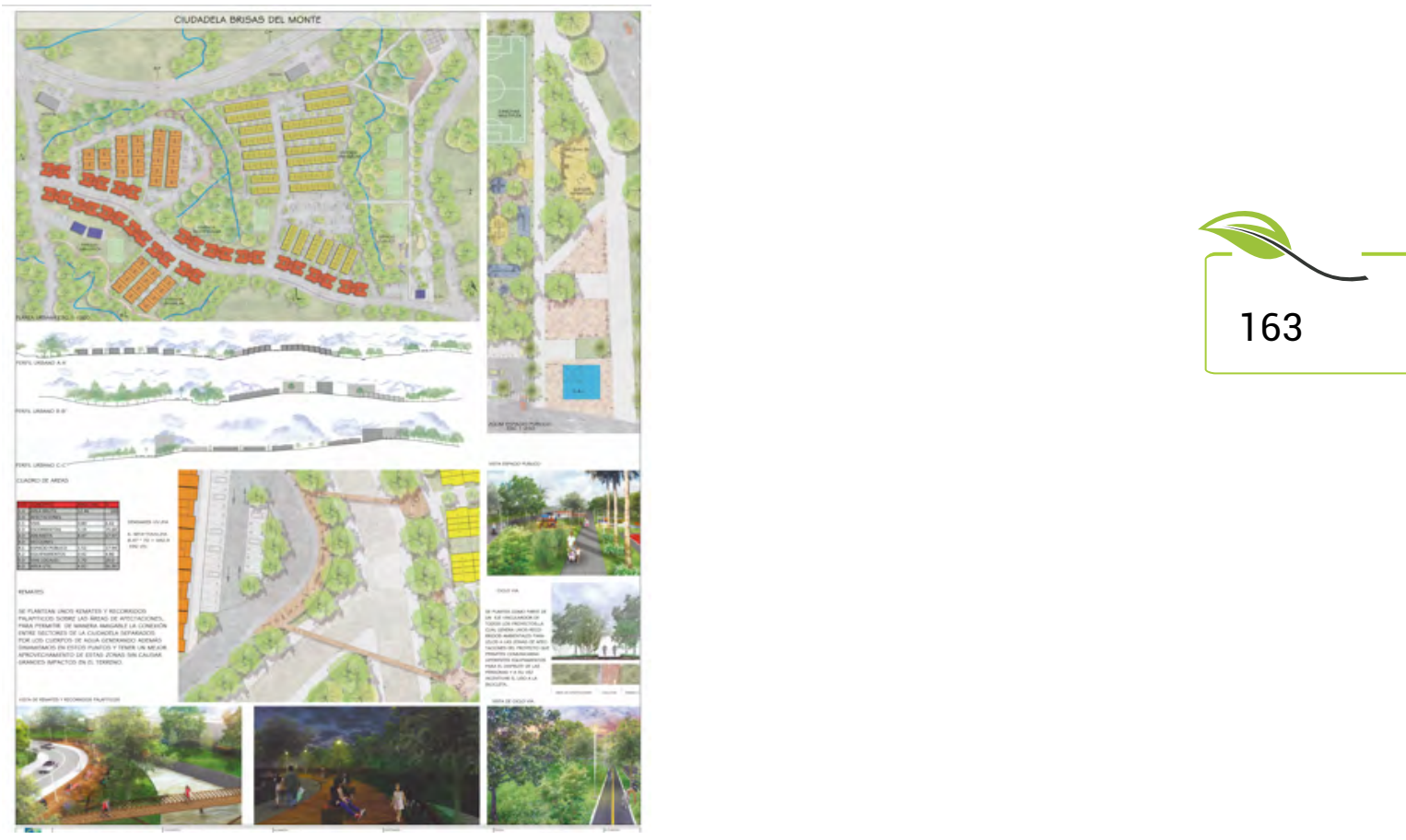

Nota. Fuente: Taller vivienda social - Programa de Arquitectura. Universidad del Pacífico. (2018).

Por consiguiente, haber densificado estos vacíos urbanos, mediante un parámetro de 50 viv/ha a 144 vivda/ha, donde el factor final terminó siendo el máximo; significó la optimización de un área urbana que, bajo las condiciones normativas actuales, representa un potencial de menor número de viviendas, que en la relación costo beneficio de la factibilidad de un proyecto terminaría siendo un factor negativo. Demostrándose, que mediante la metodología empleada en este estudio (Rodríguez y Rivero, 2017), se determinaron las densidades ideales a las condiciones particulares que se necesita; permitiendo que a futuro estas actuales zonas urbanas, sean la oportunidad precisa del proceso de densificación, que demandará un reto político de la planeación de la ciudad (Dunning et al. 2020). 
Se demostró, que no es necesario tener 6 factores de densidad como actualmente se reglamentan en el P.O.T (Acuerdo 03 de 2001 [Concejo Municipal de Buenaventura]. Por el cual se adopta el Plan de Ordenamiento Territorial para el municipio de Buenaventura. 08 de mayo de 2001), algo en la práctica muy innecesario, ya que las características de los vacíos urbanos son iguales; por lo tanto los resultados de este estudio, demostraron que los mismos se pueden simplificar solo en 2 factores (mínima y máxima) para todo el territorio, de acuerdo a la metodología empleada (Rodríguez y Rivero, 2017).

\section{Conclusiones.}

Los marcos normativos, a veces establecen unos parámetros sin saber el porqué de sus fundamentos, tal como ocurre con el P.O.T vigente; el cual, al constituir las densidades en los usos del suelo, no estableció claro los argumentos, que debieron estipularse de acuerdo a las características propias del territorio. Por lo tanto, este estudio demostró que los soportes para las densidades propuestas y simplificadas, obedecieron a las condiciones locativas y normativas, ideales para el Distrito de Buenaventura; donde los factores de las mismas, fueron determinantes para establecer las viviendas de más, que se necesitan dentro de las pocas áreas urbanas disponibles, para hacer proyectos habitacionales sociales y por ende generar una ciudad compacta y sostenible (ver Figura 9).

Por eso la importancia de esta publicación, ya que es una oportunidad de demostrar que, desde la academia se está pensado de manera objetiva e integral el desarrollo del crecimiento urbano y el problema del hábitat desde la perspectiva de la gestión de la vivienda social, como un ejercicio de planificación urbana. Pero agregándole el concepto actual de ciudad sostenible, que siempre propenderá por el ahorro de recursos y energía; tal como se demostró, al optimizar en unas zonas urbanas, la disponibilidad de más unidades habitacionales con la misma cobertura de infraestructura existente.

Infraestructura, representada en sistemas estructurantes de ciudad, que trasversalmente sirven tanto para los hábitats existentes; como para los nuevos propuestos de manera densificada, en un proceso integral de regeneración urbana. Los existentes deberán tener un enfoque de gestión cualitativa, a través de procesos de mejoramientos integrales de barrios; mientras que los nuevos, deberán tener un enfoque cuantitativo a través de la densificación; aprovechando el uso de mixturas, representada en la combinación de uso y tipologías edificatorias como: multifamiliar, bifamiliar y unifamiliar, dentro de cada uno de los proyectos.

Estos resultados, son un insumo importante en el corto plazo, para que desde las autoridades locales se puedan mejorar las densidades de estos territorios urbanos dispuestos para vivienda. Más aún, cuando todavía estamos en el proceso de revisión y actualización del P.O.T de Buenaventura, el cual venció en el año 2015; y por lo tanto, se hace la invitación a que las autoridades locales, responsables de las políticas de planeamiento urbano del territorio, tengan en cuenta estos resultados; para que oficialmente sean considerados como los acordes, porque cumplen con las características de la ciudad. 
Densificación en los vacíos urbanos del distrito de Buenaventura;

Para propender por una ciudad compacta y sostenible

Lerma Bonilla, Lides Leonardo

Figura 9. Modelación urbana de vivienda sobre vacíos urbanos: Proyecto $N^{\circ} 1$
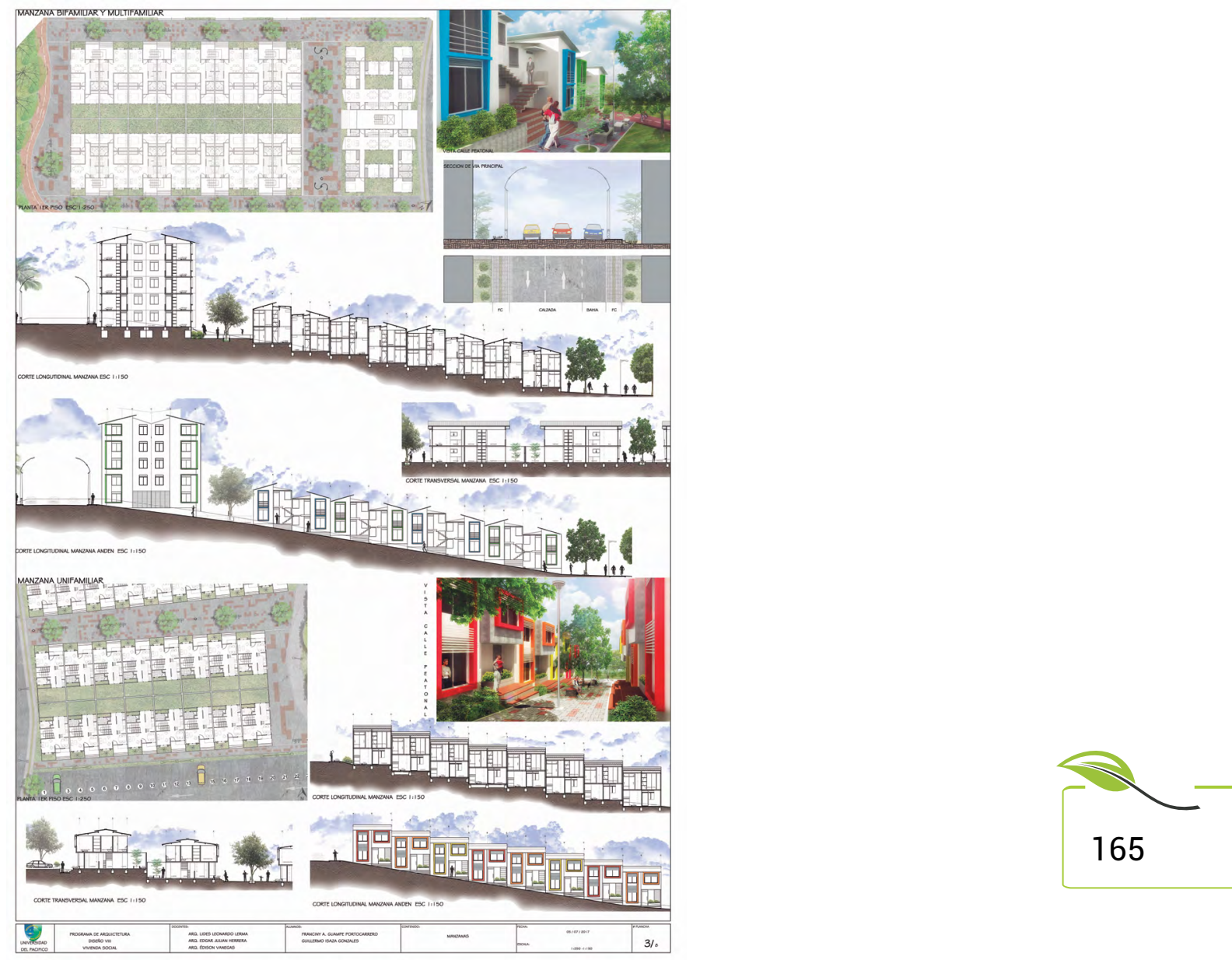

Nota. Fuente: Taller vivienda social - Programa de Arquitectura. Universidad del Pacífico. (2018). 


\section{Referencias bibliográficas}

Acuerdo $N^{\circ} 03$ de 2001. [Concejo Municipal de Buenaventura]. Por el cual se adopta el Plan de Ordenamiento Territorial para el municipio de Buenaventura, Valle del Cauca. 08 de mayo de 2001. https://www.buenaventura.gov.co/articulos/plan-de-ordenamiento-territorial

Botero, H. (1968). CVC Estudio de desarrollo y planeamiento urbano Buenaventura. C.V.C, 1-225. https://ecopedia.cvc.gov.co/categorias/costa-pacifica

Bustos, M. (2019). Regeneración habitacional urbana como herramienta para abordar la desigualdad territorial. Revista Universidad Politécnica de Cataluña, (pp.12-42). https://upcommons.upc.edu/ bitstream/handle/2117/178112/QRU9_Monica Bustos .pdf?sequence=1\&isAllowed=y

Calle, F.; Salazar, X. y González, M. (2019). La integración de aprendizajes en la enseñanza de la planificación urbana articulada a contextos reales: Evaluación del proceso pedagógico en la formación del arquitecto a través de un caso de estudio. Arquitecturas del Sur, 37(56), 100-119. https://doi.org/ 10.22320/07196466.2019.37.056.07

Carrasco, J. (2020). Supermanzana Chillaneja: regeneración urbana desde la movilidad y densificación. [Tesis de maestría, Pontifica Universidad Católica de Chile]. https://repositorio.uc.cl/ handle/11534/28518

Cuadros, M.A. (2019). Zona continental de Buenaventura. [Fotografía]. https://www.facebook.com/ michaelandres.cuadroalvarez/photos

Departamento Adminstrativo Nacional de Estadística - DANE. (2018). Censo nacional de población y vivienda - CNPV 2018. https://www.dane.gov.co/index.php/estadisticas-por-tema/demografia-ypoblacion/censo-nacional-de-poblacion-y-vivenda-2018

Debrunner, G., Hengstermann, A. y Gerber, J. D. (2020). The business of densification: Distribution of power, wealth and inequality in swiss policy making. Town Planning Review, 91 (3), (pp. 259-281). https://doi.org/10.3828/tpr.2020.15

Dunning, R.; Hickman, H. y While, A. (2020). Planning control and the politics of soft densification. Town Planning Review, 91(3), (pp. 305-324). https://doi.org/10.3828/tpr.2020.17

Elvestad, H. y Holsen, T. (2020). Negative covenants and real-estate developers' modus operandi: The case of suburban densification in oslo, Norway. Town Planning Review, 91(3), (pp. 325-342). https:// doi.org/10.3828/tpr.2020.18

Escallón, C. (2011). La vivienda de interés social en Colombia , principios y retos. Revista de Ingeniería Universidad de Los Andes, 35, (pp. 55-60). https://www.redalyc.org/articulo.oa?id=121022763010

García, F. (2019). Los valores de compacidad y densidad en los tejidos residenciales de la periferia de las ciudades intermedias españolas a lo largo del siglo XX. ACE 40, 14 (40), (pp. 11-34). https:// upcommons.upc.edu/handle/2117/165285

Gómez, L. (2018). Cambios de usos de suelo en Paso de los Libres, Corrientes, Argentina entre 1990 y 2016. Impacto del proceso de expansión urbana. Revista Geográfica Digital, (pp. 1-21). https://hum. unne.edu.ar/revistas/geoweb/default.htm

Gómez. y Mesa, A. (2017). Determinación de densidades urbanas sostenibles en base a metodología relativa al acceso solar : caso área metropolitana de Mendoza, Argentina. Revista de Urbanismo, (pp. 131-145). https://revistaurbanismo.uchile.cl/ 
Gonzales, Á. (2018). Urbanismo educativo y discapacidad. Nuevos mecanismos participativos para una ciudad más sostenible e integradora. El caso del plan maestro del centro histórico del D.C. de Honduras. Arquitecturas del Sur, 36, (pp. 84-93). http://revistas.ubiobio.cl/index.php/AS/article/ view/3301

Granados, A. (2019). La expansión urbana como centro de tensión entre la normativa liberalizadora y la planificación urbana en Perú (1995-2019). Revista Ciudades, Estado y Política, 5 (3), (pp. 37-55). https://revistas.unal.edu.co/index.php/revcep/article/view/82185

Jiménez, C. (2015). Análisis de la ocupación de suelo por las áreas urbanas españolas Tamaño y densidad urbana. [Tesis de doctorado, Universidad Politécnica de Madrid]. http://habitat.aq.upm.es/ tydu/tydu.pdf

Lerma, L. (2019). Análisis administrativo y técnico, del modelo de gestión pública, para la construcción de vivienda de interés social en el distrito especial de Buenaventura: estudio de caso. [Tesis de maestria, Pontificia Universidad Javeriana]. http://vitela.javerianacali.edu.co/handle/11522/12120

Ley 185 de 1959. Por la cual se ordena el estudio de algunas obras en el Municipio de Buenaventura y la costa vallecaucana del Pacífico, y se conceden facultades al Gobierno para tal fin. 30 de diciembre de 1959. Diario Oficial No. 30.140. http://www.suin-juriscol.gov.co/viewDocument. asp?ruta=Leyes/1654349

Ley 388 de 1997. Por medio del cual se modifica la Ley 9 de 1989, y la Ley 2 de 1991 y se dictan otras disposiciones. 18 de Julio de 1997. Diario Oficial No. 43.091. http://www.suin-juriscol.gov.co/ viewDocument.asp?ruta=Leyes/1654349

Libertun de Duren, N. (2017). Los motivos por los que promotores privados de vivienda social construyen en las periferias de las ciudades de américa latina. Banco Interamericano de Desarrollo, 1-31. htpp://www.iadb.org

Montrezor, D. y Bernardini, S. (2019). Planejamento e desenho urbanos : uma conciliação possível? Revista Basileira de Gestao Urbana, 1-34. https://doi.org/10.1590/2175-3369.011.e20180133

Pérez, M. (2019). Hogares unipersonales y curso de vida: diversificación por edades y concentración espacial en las regiones. Estudios Geográficos, 80 (287), (pp.1-19). http://estudiosgeograficos. revistas.csic.es/index.php/estudiosgeograficos/article/view/750

Piña, E. (2018). Vertical social sustentable, enfoque en sustainable social high-rise housing, an approach to climate change resilience 1 resumen. Revista Invi, 33(92), (pp. 213-237). https://scielo. conicyt.cl/scielo.php?script=sci_arttext\&pid=S0718-83582018000100213

Ramos, J. (2017). Propuesta metodológica para determinar la sostenibilidad urbana de San Juan de Pasto bajo la perspectiva de entropía urbana. [Tesis de maestría, Universidad Distrital Francisco José de Caldas]. http://sie.car.gov.co/handle/11349/6978

Remesar, A. (2019). Del arte público al post-muralismo. Revista Científica de La Universidad de Barcelona, 61, (pp. 3-65). https://revistes.ub.edu/index.php/waterfront/article/view/28647/0

Rodrígues, P. (2018). El desarrollo de los suburbios latinoamericanos bajo nuevos paradigmas. Reflexiones sobre el ejemplo de Jundiaí, Brasil. Arquitecturas del Sur, 36(54), (pp. 20-31). https:// doi.org/10.22320/07196466.2018.36.054.02

Rodríguez, J. y Rivero, F. (2017). Calculation of housing density for a compact city. Application to the city of Sevilla. Revista Científica Monfragüe, 1-13. https://www.eweb.unex.es/eweb/monfragueresilente/ 
Sierra, F. (2018). Evaluación de la accesibilidad y la distribución de parques públicos urbanos en Bogotá. Pontificia Universidad Javeriana, (pp.1-29). https://repository.javeriana.edu.co/handle/10554/40268

Treija, S., Bratuškins, U. y Korolova, A. (2019). Urban Densification of Large Housing Estates in the Context of Privatisation of Public Open Space: the Case of Imanta, Riga. Architecture and Urban Planning, 14(1), (pp. 105-110). https://doi.org/10.2478/aup-2018-0014

Vergara, J. y Asenjo, D. (2019). La transformación distinta a la forma. Contingencias no arquitectónicas sobre edificios en altura en la prensa chilena (2009-2017). Arquitecturas del Sur, 37(55), (pp. 92-105). https://doi.org/10.22320/07196466.2019.37.055.06

Yunda, J. y Montenegro, G. (2019). Cualidades del paisaje construido de la vivienda y su impacto en la morfología y densidades de Bogotá. Revista Invi, 34 (August), (pp. 105-126). https://scielo.conicyt. cl/scielo.php?script=sci_arttext\&pid=S0718-83582019000200105

Zumelsu, A. y Espinoza, D. (2019). Elaboración de una metodología para evaluar sostenibilidad en barrios de ciudades intermedias en Chile. Revista 180, (pp. 80-94). https://scielo.conicyt.cl/scielo. php?pid=S0718-669X2019000200080\&script=sci_arttext

Lerma Bonilla, Lides Leonardo Universidad del Pacífico https://orcid.org/ 0000-0001-5089-942X

Es docente del Programa de Arquitectura de la Universidad del Pacífico; Arquitecto 2001 y Master en Ingeniería Civil, énfasis en Construcciones 2019; dirige el taller de diseño 7: Vivienda Social.

Email: Illerma@unipacifico.edu.co. 
ANEXO 1.

Figura 10. Descripción superficie vial (Svia) de Buenaventura

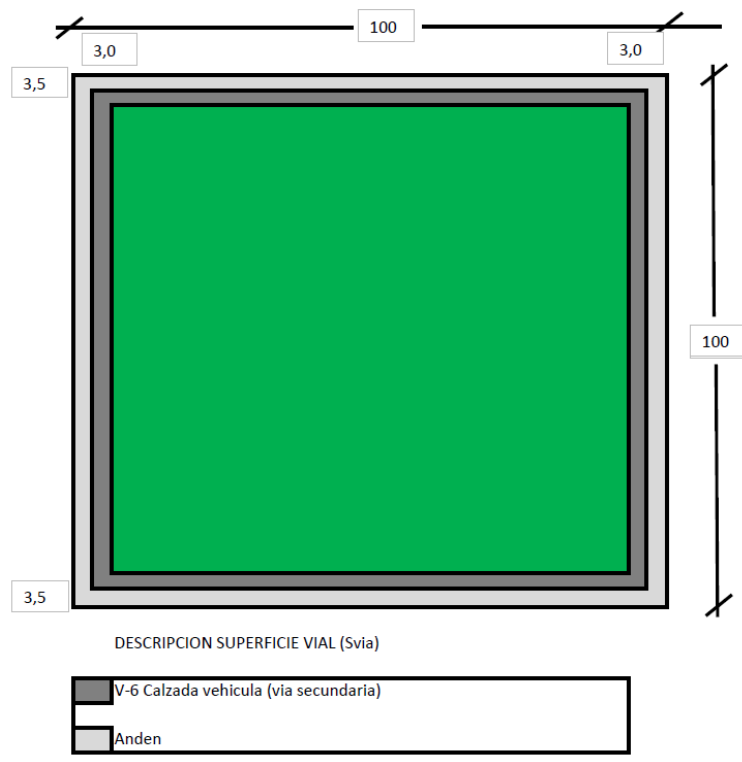

ANEXO 2.

Tabla $\mathrm{N}^{\circ} 3$

DESCRIPCION DE LOS 11 PROYECTOS DE MODELACION URBANA

\begin{tabular}{|c|c|}
\hline $\begin{array}{l}\text { Coordinador } \\
\text { Docentes }\end{array}$ & $\begin{array}{l}\quad \text { Universidad del Pacifico - Programa de Arquiectura } \\
\qquad \text { Diseño 7: Taller Vivienda Social } \\
\text { Lides Leonardo Lerma Bonilla } \\
\text { Lides Leonardo Lerma Bonilla } \\
\text { Edgar Julian Herera Aguilar }\end{array}$ \\
\hline PROYECTO 1 & $\begin{array}{l}\text { Proyecto presentado por los alumnos Franciny Guape y Guillermo Isaza, ubicado al costado norte de la } \\
\text { ciudad, barrio Doña Cesi, entre la via alterna interna y la Univesidad del Valle }\end{array}$ \\
\hline PROYECTO 2 & $\begin{array}{l}\text { Proyecto presentado por los alumnos Emerson Estupiñan y Albeiro Cabezas, ubicado al costadosur de la } \\
\text { ciudad, barrio Antonio Nariño, en el estero San Antonio }\end{array}$ \\
\hline PROYECTO 3 & $\begin{array}{l}\text { Proyecto presentado por los alumnos Angie Perlaza y Karina Angulo, ubicado al costado sur de la ciudad, } \\
\text { margen izquierda via al aeropuerto, cerca a la quebrada Mondomo }\end{array}$ \\
\hline PROYECTO 4 & $\begin{array}{l}\text { Proyecto presentado por los alumnos Milady Mina y Michael Cifuentes, ubicado al costado sur de la ciudad, } \\
\text { barrio el triunfo }\end{array}$ \\
\hline PROYECTO 5 & $\begin{array}{l}\text { Proyecto presentado por los alumnos Owar Riascos y Nelly Lopez, ubicado al costado sur de la ciudad, } \\
\text { barrio el triunfo }\end{array}$ \\
\hline PROYECTO 6 & $\begin{array}{l}\text { Proyecto presentado por los alumnos Victor Bermudez y Vivivana Perez, ubicado al costado sur de la } \\
\text { ciudad, barrio el triunfo }\end{array}$ \\
\hline PROYECTO 7 & $\begin{array}{l}\text { Proyecto presentado por los alumnos Kevin Satizabal y Edinson Quiñonez, ubicado al costado sur de la } \\
\text { ciudad, barrio el triunfo }\end{array}$ \\
\hline PROYECTO 8 & $\begin{array}{l}\text { Proyecto presentado por los alumnos Maria Linares, Eovin Piedrahita, Jhoisir, en el sector de la via Cabal } \\
\text { pombo cerca a Cittronela }\end{array}$ \\
\hline PROYECTO 9 & $\begin{array}{l}\text { Proyecto presentado por los alumnos Gerson Rendon y Yaritze, ubicado al costado sur de la ciudad,barrio } \\
\text { Las Palmas }\end{array}$ \\
\hline PROYECTO 10 & $\begin{array}{l}\text { Proyecto presentado por los alumnos Gustavo Cajigas y Cristian Sanchez, ubicado al costado sur de la } \\
\text { ciudad,barrio Las Palmas }\end{array}$ \\
\hline PROYECTO 11 & $\begin{array}{l}\text { Proyecto presentado por los alumnosBryan Caicedo y Sara, ubicado al costado sur de la ciudad,barrio Las } \\
\text { Palmas }\end{array}$ \\
\hline
\end{tabular}

Descripción de los 11 anteproyectos de modelación urbana

Nota. Fuente: Taller vivienda social - Programa de Arquitectura. Universidad del Pacífico. (2018). 